\title{
Numerical Solution of System of Three Nonlinear Volterra Integral Equations Using Implicit Trapezoidal
}

\author{
Dalal Adnan Maturi ${ }^{1}$ \& Honaida Mohammed Malaikah ${ }^{2}$ \\ ${ }^{1}$ Departement of Mathematics, Faculty of Science, King Abdulaziz University,Jeddah, Saudi Arabia \\ ${ }^{2}$ Departement of Mathematics, Faculty of Science, King Abdulaziz University, Jeddah, Saudi Arabia \\ Correspondence: Dalal Adnan Maturi, Departement of Mathematics, Faculty of Science, King Abdulaziz University, \\ P.O.Box 42664, Jeddah 21551, Saudi Arabia. E-mail: maturi_dalal2020@yahoo.com
}

Received: November 15, 2017 Accepted: December 1, 2017 Online Published: December 13, 2017

doi:10.5539/jmr.v10n1p44 URL: https://doi.org/10.5539/jmr.v10n1p44

\begin{abstract}
In this project, we will be find numerical solution of Volterra Integral Equation of Second kind through using Implicit trapezoidal and that by using Maple 17 program, then we found that numerical solution was highly accurate when it was compared with exact solution.
\end{abstract}

Keywords: volterra integral equation of second kind, implicit trapezoidal method, nonlinear programing

\section{Introduction}

We consider the Volterra integral equation of the second kind

$$
x(t)=f(t)+\int_{0}^{t} k(t, s, x(s)) d s
$$

Where $x, f$ and $k$ are vector-valued functions with $m$ components. If $f$ and $k$ are continuous and $k(t, s, x(s))$ satisfies a Lipcshitz condition with respect to $x$, then a unique solution $x(t)$ of (1) exists (Balakumar, V. \& Murugesan, K., 2013; Berenguer, M. I. \& et al., 2011; Burton,T. A., 2005).

Volterra integral equations have been found to be effective to describe some application such as potential theory and Dirichlet problemsand electrostatics. Also, Volterra integral equations areapplied in the biology, chemistry, engineering, mathematical problems of radiation equilibrium,theparticle transport problems of astrophysics and reactor theory, and radiation heat transfer problems (Balakumar, V. \& Murugesan, K., 2013; Maturi, D. A., 2014).

The systems of Volterra integral equations appear in two kinds. For systems of Volterra integral equations of the first kind, the unknown functions appear only under the integral sign in the form:

$$
\begin{aligned}
& f_{1}(t)=\int_{0}^{t}\left(K_{1}(t, s) u(s)+\widetilde{K_{1}}(t, s) v(s)+\cdots\right) d s \\
& f_{2}(t)=\int_{0}^{t}\left(K_{2}(t, s) u(s)+\widetilde{K_{2}}(t, s) v(s)+\cdots\right) d s,
\end{aligned}
$$

However, systems of Volterra integral equations of the second kind, the unknown functions appear inside and outside the integral sign of the form:

$$
\begin{aligned}
& u(t)=f_{1}(t)+\int_{0}^{t}\left(K_{1}(t, s) u(s)+\widetilde{K_{1}}(t, s) v(s)+\cdots\right) d s \\
& v(t)=f_{2}(t)+\int_{0}^{t}\left(K_{2}(t, s) u(s)+\widetilde{K_{2}}(t, s) v(s)+\cdots\right) d s,
\end{aligned}
$$


The kernels $K_{i}(t, s)$ and $\widetilde{K}_{l}(t, s)$, and the functions $f_{i}(t), i=1,2, \cdots, n$ are given real-valued functions.

A variety of analytical and numerical methods are used to handle systems of Volterra integral equations. The existing techniques encountered some difficulties in terms of the size of computational work, especially when the system involves several integral equations (Linz, P., 1985).

In this project, we present the computation of numerical solution of systems of Volterra integral equation of the second kind.

\section{Preliminaries}

In this section, we recall the main theorems (Effati, S. \& NooriSkandari, M. H., 2012).

Theorem 1.Consider the equation

$$
x(t)=f(t)+\int_{0}^{t} p(t, s) k(t, s) x(s) d s
$$

Where

1) $f(t)$ is continuous in $0 \leq t \leq T$.

2) $k(t, s)$ is a continuous function in $0 \leq s \leq t \leq T$,

3) for each continuous function $h$ andall

$0 \leq \tau_{1} \leq \tau_{2} \leq t$ the integrals

$$
\begin{aligned}
& \int_{\tau_{1}}^{\tau_{2}} p(t, s) k(t, s) h(s) d s \\
& \int_{0}^{t} p(t, s) k(t, s) h(s) d s
\end{aligned}
$$

are continuous functions of $t$,

4) $p(t, s)$ is absolutely integrable with respect to $s$ for all $0 \leq t \leq T$,

5) there exist points $0=T_{0}<T_{1}<T_{2}<\cdots<T_{N}=T$ such that with $t \geq T_{1}$

$$
k \int_{T_{i}}^{\min \left(t, T_{i+1}\right)}|p(t, s)| d s \leq \alpha<1,
$$

Were $k=\max _{0 \leq s \leq t \leq T}|k(t, s)|$,

6) for every $t \geq 0$ such that with $t \geq T_{1}$

$$
\lim _{\delta \rightarrow 0^{+}} \int_{t}^{t+\delta}|p(t+\delta, s)| d s=0 .
$$

Then (2) has a unique continuous solution in $0 \leq t \leq T$.

Theorem 2.Consider the equation

$$
x(t)=f(t)+\int_{0}^{t} p(t, s) k(t, s, x(s)) d s
$$

Where

1) $f(t)$ is continuous in $0 \leq t \leq T$.

2) $k(t, s, u)$ is a continuous function in $0 \leq s \leq t \leq T,-\infty<u<\infty$,

3) the Lipschitz condition

$$
|k(t, s, y)-k(t, s, z)| \leq L|y-z|
$$


is satisfies for $0 \leq s \leq t \leq T$ and all $y$ and $z$,

4) $p(t, s)$ satisfies conditions (3)-(4) of Theroem 1 with $k$ replaced by $L$ and $k(t, s, h(s)$ ) instead of $k(t, s) h(s)$.

Then (3) has a unique continuous solution in $0 \leq t \leq T$.

\section{The Mathematics of the Volterra Procedure}

In this section, we use the technique of the Volterra equation (Balakumar, V. \& Murugesan, K., 2013; Effati, S. \& NooriSkandari, M. H., 2012) to find an approximates the solution $x(t)$ of (1) at the equally spaced points $t_{n}=t_{0}+n h$ for $n=1, \cdots, N$ where $t_{0}=0$ and $N$ is the total number of steps of size $h . X_{n}$ denotes the approximation of $x(t)$ at $t=t_{n}$.

Setting $t=t_{n}$ in (1), we have

$$
x\left(t_{n}\right)=f\left(t_{n}\right)+\int_{0}^{t_{n}} k\left(t_{n}, t, x(t)\right) d t
$$

By the composite trapezoidal rule an approximation of the integral in (4) is

$$
\frac{h}{2}\left[k\left(t_{n}, t_{0}, x\left(t_{0}\right)\right)+2 \sum_{j=1}^{n-1} k\left(t_{n}, t_{j}, x\left(t_{j}\right)\right)+k\left(t_{n}, t_{n}, x\left(t_{n}\right)\right)\right]
$$

Replacing $x\left(t_{n}\right)$ in (4) and (5) by $X_{n}$, we obtain the implicit trapezoidal rule

$$
X_{n}=f\left(t_{n}\right)+h\left[\frac{1}{2} k\left(t_{n}, t_{0}, X_{0}\right)+\sum_{j=1}^{n-1} k\left(t_{n}, t_{j}, X_{j}\right)+\frac{1}{2} k\left(t_{n}, t_{n}, X_{n}\right)\right]
$$

Where $X_{0}=f(0)$ since $x(0)=f(0)$.

Defining $\sigma_{n}$ by

$$
\sigma_{n}=f\left(t_{n}\right)+h\left[\frac{1}{2} k\left(t_{n}, t_{0}, X_{0}\right)+\sum_{j=1}^{n-1} k\left(t_{n}, t_{j}, X_{j}\right)\right]
$$

We can rewrite (6) as

$$
X_{n}-\frac{1}{2} h k\left(t_{n}, t_{n}, X_{n}\right)-\sigma_{n}=0,
$$

Where 0denotes the zero vector. From (8), we see that $X_{n}$ is the solution of the vector equation

Where $\phi$ is the vector-valued function

$$
\phi(u)=0,
$$

$$
\phi(u)=u-\frac{1}{2} h k\left(t_{n}, t_{n}, u\right)-\sigma_{n}
$$

We will obtain an approximation to the solution $X_{n}$ of (9) by way of the matrix-valued function $G$ defined in (11). If $A(u)$ is an $m$ by $m$ matrix-valued function that is invertible in a neighborhood of $X_{n}$, then $X_{n}$ is a fixed point of

$$
G(u)=u-A(u) \phi(u) .
$$

Assuming the components of $G(u)$ have continuous first and second order partial derivatives and that the first order partial derivatives and that the first order partial derivatives at

$X_{n}$ are equal to zero, it can be shown that if $A(u)$ is set equal to the Jacobian matrix of the function $\phi$, the iterates $X_{n}^{(p)}$ defined by (13) below will usually converge quadratically to $X_{n}$ provided the starting value is sufficiently close to $X_{n}$. The Jacobian matrix of $\phi$ is the $m$ by $m$ matrix $J(u)$ with the element

$$
J(u)_{i j}=\frac{\partial}{\partial u_{j}} \phi_{i}(u)=\delta_{i j}-\frac{1}{2} h \frac{\partial}{\partial u_{j}} k_{i}\left(t_{n}, t_{n}, u\right)
$$

In row $i$ and column, where $\delta_{i j}$ is the Kronecker delta. Details of the statements made here follow from the discussion of Newton's method for nonlinear systems in (Balakumar, V. \& Murugesan, K., 2013). Linz gives a brief outline of the trapezoidal rule and Newton's method for Volterra integral systems of the second kind in Section of (Effati, S. \& NooriSkandari, M. H., 2012). 
We obtain $X_{n}$ from $X_{n-1}$ by setting $X_{n}^{(0)}=X_{n-1}$ and then generating the iterates $X_{n}^{(p)}$ from

$$
X_{n}^{(p)}=G\left(X_{n}^{(p-1)}\right)=X_{n}^{(p-1)}-J^{-1}\left(X_{n}^{(p-1)}\right) \phi\left(X_{n}^{(p-1)}\right)
$$

For $p=1,2,3, \cdots$. (This is Newton's method for nonlinear systems.) Let $y$ denote the solution of the matrix equation

$$
J\left(X_{n}^{(p-1)}\right) y=\phi\left(X_{n}^{(p-1)}\right)
$$

Then the iteration formula (13) becomes

$$
X_{n}^{(p)}=X_{n}^{(p-1)}-y
$$

We compute the solution $y=J^{-1}\left(X_{n}^{(p-1)}\right) \phi\left(X_{n}^{(p-1)}\right)$ using the command Linear Solve. The iterates $X_{n}^{(p)}$ are computed until the infinity norm of the vector $y$ is less than a prescribed tolerance Tol. Then $X_{n}$ is assigned the value of the last iterate (Balakumar, V. \& Murugesan, K., 2013; Effati, S. \& NooriSkandari, M. H., 2012).

\section{Numerical Example}

In this section, we solve some examples, and we can compare the numerical results with the exact solution.

Example1. Consider the system of Volterra integral equations

$$
\begin{gathered}
\mathrm{X}_{1}(t)=1-t^{2}+\frac{1}{4} t^{4}+\int_{0}^{t}\left(\mathrm{X}_{1}(s)+\mathrm{X}_{2}(s)-\mathrm{X}_{3}(s)\right) d s \\
\mathrm{X}_{2}(t)=2 t+t^{2}-\frac{2}{3} t^{3}-\frac{1}{4} t^{4}+\int_{0}^{t}\left(\mathrm{X}_{2}(s)+\mathrm{X}_{3}(s)-\mathrm{X}_{1}(s)\right) d s \\
\mathrm{X}_{3}(t)=-t+t^{2}+t^{3}-\frac{1}{4} t^{4}+\int_{0}^{t}\left(\mathrm{X}_{3}(s)+\mathrm{X}_{1}(s)-\mathrm{X}_{2}(s)\right) d s
\end{gathered}
$$

With the exact solution $\mathrm{X}_{1}(t)=1+t, \mathrm{X}_{2}(t)=t+t^{2}$ and $\mathrm{X}_{3}(t)=t^{2}+t^{3}$.

Table.1 Numerical results and exact solution of systems of three Nonlinear Volterra integral equations for example 1.

\begin{tabular}{ccccccc}
\hline $\boldsymbol{t}$ & $\boldsymbol{X}_{\mathbf{1}}(\boldsymbol{t})$ & $\boldsymbol{X}_{\mathbf{2}}(\boldsymbol{t})$ & $\boldsymbol{X}_{\mathbf{3}}(\boldsymbol{t})$ & $\begin{array}{c}\text { Exact1 } \\
=\mathbf{1}+\boldsymbol{t}\end{array}$ & $\begin{array}{c}\text { Exact2 } \\
=\boldsymbol{t}+\boldsymbol{t}^{\mathbf{2}}\end{array}$ & $\begin{array}{c}\text { Exact3 } \\
=\boldsymbol{t}^{2}+\boldsymbol{t}^{\mathbf{3}}\end{array}$ \\
\hline 0.00 & 1.0000 & 0.0000 & 0.0000 & 1.0000 & 0.0000 & 0.0000 \\
0.05 & 1.0500 & 0.0525 & 0.0026 & 1.0500 & 0.0525 & 0.0026 \\
0.10 & 1.1000 & 0.1100 & 0.0110 & 1.1000 & 0.1100 & 0.0110 \\
0.15 & 1.1500 & 0.1725 & 0.0259 & 1.1500 & 0.1725 & 0.0259 \\
0.20 & 1.2000 & 0.2400 & 0.0480 & 1.2000 & 0.2400 & 0.0480 \\
0.25 & 1.2500 & 0.3125 & 0.0781 & 1.2500 & 0.3125 & 0.0781 \\
0.30 & 1.3000 & 0.3900 & 0.1170 & 1.3000 & 0.3900 & 0.1170 \\
0.35 & 1.3500 & 0.4725 & 0.1654 & 1.3500 & 0.4725 & 0.1654 \\
0.40 & 1.4000 & 0.5600 & 0.2240 & 1.4000 & 0.5600 & 0.2240 \\
0.45 & 1.4500 & 0.6525 & 0.2936 & 1.4500 & 0.6525 & 0.2936 \\
0.50 & 1.5000 & 0.7500 & 0.3750 & 1.5000 & 0.7500 & 0.3750 \\
0.55 & 1.5500 & 0.8525 & 0.4689 & 1.5500 & 0.8525 & 0.4689
\end{tabular}




\begin{tabular}{lllllll}
0.60 & 1.6000 & 0.9600 & 0.5760 & 1.6000 & 0.9600 & 0.5760 \\
0.65 & 1.6500 & 1.0725 & 0.6971 & 1.6500 & 1.0725 & 0.6971 \\
0.70 & 1.7000 & 1.1900 & 0.8330 & 1.7000 & 1.1900 & 0.8330 \\
0.75 & 1.7500 & 1.3126 & 0.9844 & 1.7500 & 1.3125 & 0.9844 \\
0.80 & 1.8000 & 1.4401 & 1.1520 & 1.8000 & 1.4400 & 1.1520 \\
0.85 & 1.8500 & 1.5726 & 1.3366 & 1.8500 & 1.5725 & 1.3366 \\
0.90 & 1.9000 & 1.7101 & 1.5390 & 1.9000 & 1.7100 & 1.5390 \\
0.95 & 1.9500 & 1.8526 & 1.7599 & 1.9500 & 1.8525 & 1.7599 \\
1.00 & 2.0000 & 2.0001 & 2.0000 & 2.0000 & 2.0000 & 2.0000 \\
1.05 & 2.0500 & 2.1526 & 2.2601 & 2.0500 & 2.1525 & 2.2601 \\
1.10 & 2.1000 & 2.3101 & 2.5410 & 2.1000 & 2.3100 & 2.5410 \\
1.15 & 2.1500 & 2.4726 & 2.8434 & 2.1500 & 2.4725 & 2.8434 \\
1.20 & 2.2000 & 2.6401 & 3.1680 & 2.2000 & 2.6400 & 3.1680 \\
1.25 & 2.25000 & 2.8126 & 3.5156 & 2.2500 & 2.8125 & 3.5156 \\
1.30 & 2.3000 & 2.9901 & 3.8870 & 2.3000 & 2.9900 & 3.8870 \\
1.35 & 2.3500 & 3.1726 & 4.2828 & 2.3500 & 3.1725 & 4.2829 \\
1.40 & 2.4001 & 3.3602 & 4.7040 & 2.4000 & 3.3600 & 4.7040 \\
1.45 & 2.4501 & 3.5527 & 5.1511 & 2.4500 & 3.5525 & 5.1511 \\
1.50 & 2.5001 & 3.7502 & 5.6250 & 2.5000 & 3.7500 & 5.6250 \\
\hline
\end{tabular}



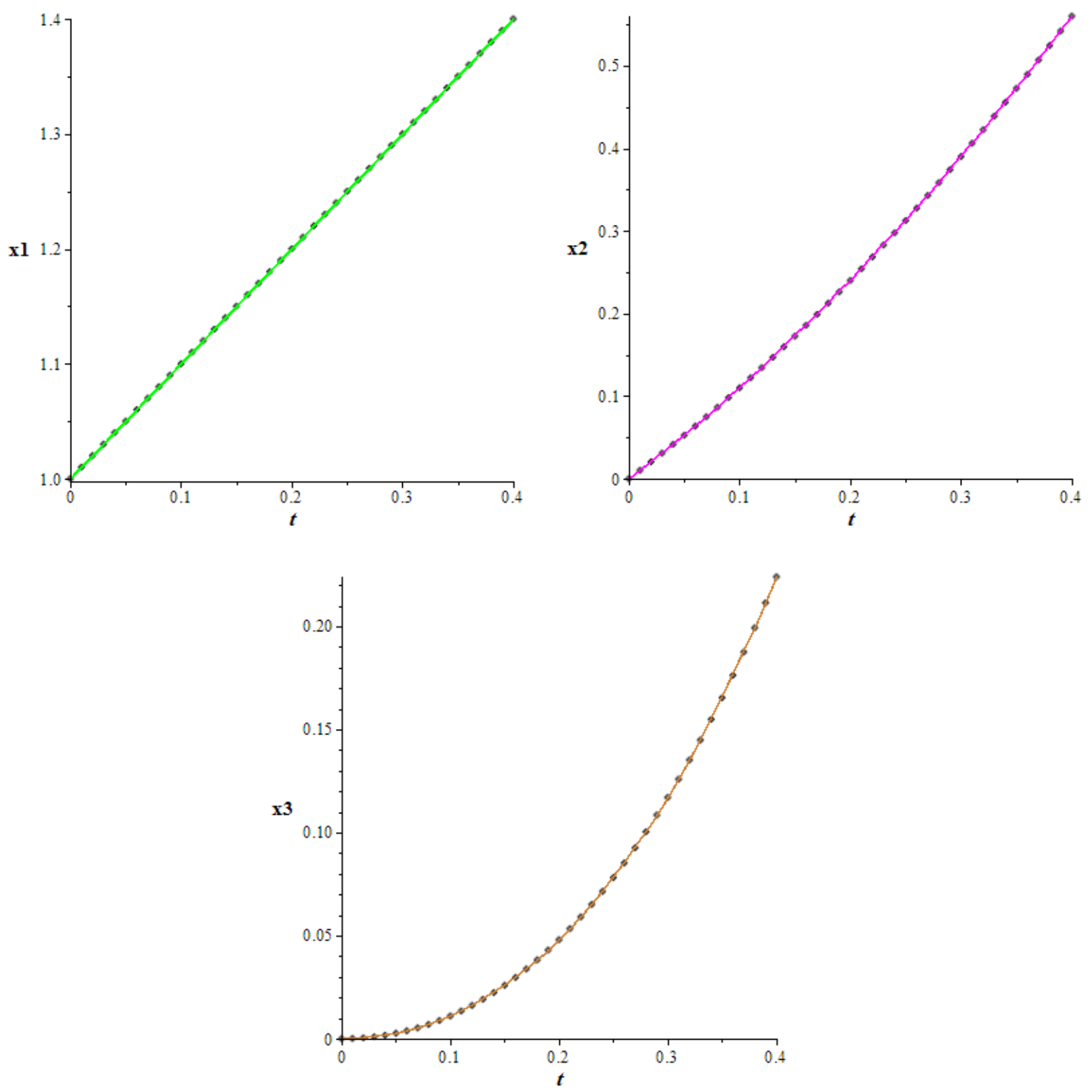

Fig. 1 The exact and approximate solutions result of systems of three Nonlinear Volterra integral equations for example 1.

Example2. Consider the system of Volterra integral equations

$$
\begin{gathered}
\mathrm{X}_{1}(t)=\cos t-\sin t-1+\int_{0}^{t}\left(\mathrm{X}_{2}(s)+\mathrm{X}_{3}(s)\right) d s \\
\mathrm{X}_{2}(t)=3 \cos t-\sin t-2+\int_{0}^{t}\left(\mathrm{X}_{1}(s)+\mathrm{X}_{3}(s)\right) d s \\
\mathrm{X}_{3}(t)=2 \cos t-1+\int_{0}^{t}\left(\mathrm{X}_{1}(s)+\mathrm{X}_{2}(s)\right) d s
\end{gathered}
$$

With the exact solution $\mathrm{X}_{1}(t)=\sin t, \mathrm{X}_{2}(t)=\cos t$ and $\mathrm{X}_{3}(t)=\sin \mathrm{t}+\cos t$. 
Table.2 Numerical results and exact solution of systems of three Nonlinear Volterra integral equations for example2.

\begin{tabular}{|c|c|c|c|c|c|c|}
\hline$t$ & $X_{1}(t)$ & $X_{2}(t)$ & $X_{3}(t)$ & $\begin{array}{l}\text { Exact } 1 \\
=\sin t\end{array}$ & $\begin{array}{l}\text { Exact } 2 \\
=\cos t\end{array}$ & $\begin{array}{l}\text { Exact } 3 \\
=\sin t \\
+\cos t\end{array}$ \\
\hline 0.00 & 0.0000 & 1.0000 & 1.0000 & 0.0000 & 1.0000 & 1.0000 \\
\hline 0.05 & 0.0500 & 0.9987 & 1.0487 & 0.0500 & 0.9988 & 1.0487 \\
\hline 0.10 & 0.0998 & 0.9950 & 1.0948 & 0.0998 & 0.9950 & 1.0948 \\
\hline 0.15 & 0.1494 & 0.9888 & 1.1382 & 0.1494 & 0.9888 & 1.1382 \\
\hline 0.20 & 0.1987 & 0.9801 & 1.1787 & 0.1987 & 0.9801 & 1.1787 \\
\hline 0.25 & 0.2474 & 0.9689 & 1.2163 & 0.2474 & 0.9689 & 1.2163 \\
\hline 0.30 & 0.2955 & 0.9553 & 1.2509 & 0.2955 & 0.9553 & 1.2509 \\
\hline 0.35 & 0.3429 & 0.9394 & 1.2823 & 0.3429 & 0.9394 & 1.2823 \\
\hline 0.40 & 0.3894 & 0.9211 & 1.3105 & 0.3894 & 0.9211 & 1.3105 \\
\hline 0.45 & 0.4350 & 0.9004 & 1.3354 & 0.4350 & 0.9004 & 1.3354 \\
\hline 0.50 & 0.4794 & 0.8776 & 1.3570 & 0.4794 & 0.8776 & 1.3570 \\
\hline 0.55 & 0.5227 & 0.8525 & 1.3752 & 0.5227 & 0.8525 & 1.3752 \\
\hline 0.60 & 0.5646 & 0.8253 & 1.3900 & 0.5646 & 0.8253 & 1.3900 \\
\hline 0.65 & 0.6052 & 0.7961 & 1.4013 & 0.6052 & 0.7961 & 1.4013 \\
\hline 0.70 & 0.6442 & 0.7648 & 1.4090 & 0.6442 & 0.7648 & 1.4091 \\
\hline 0.75 & 0.6816 & 0.7317 & 1.4133 & 0.6816 & 0.7317 & 1.4133 \\
\hline 0.80 & 0.7173 & 0.6967 & 1.4140 & 0.7174 & 0.6967 & 1.4141 \\
\hline 0.85 & 0.7512 & 0.6600 & 1.4112 & 0.7513 & 0.6600 & 1.4113 \\
\hline 0.90 & 0.7833 & 0.6216 & 1.4049 & 0.7833 & 0.6216 & 1.4049 \\
\hline 0.95 & 0.8134 & 0.5816 & 1.3951 & 0.8134 & 0.5817 & 1.3951 \\
\hline 1.00 & 0.8414 & 0.5403 & 1.3817 & 0.8415 & 0.5403 & 1.3818 \\
\hline 1.05 & 0.8674 & 0.4975 & 1.3649 & 0.8674 & 0.4976 & 1.3650 \\
\hline 1.10 & 0.8912 & 0.4535 & 1.3448 & 0.8912 & 0.4536 & 1.3448 \\
\hline 1.15 & 0.9127 & 0.4084 & 1.3212 & 0.9128 & 0.4085 & 1.3213 \\
\hline 1.20 & 0.9320 & 0.3623 & 1.2943 & 0.9320 & 0.3624 & 1.2944 \\
\hline 1.25 & 0.9489 & 0.3152 & 1.2642 & 0.9490 & 0.3153 & 1.2643 \\
\hline 1.30 & 0.9635 & 0.2674 & 1.2310 & 0.9636 & 0.2675 & 1.2311 \\
\hline 1.35 & 0.9756 & 0.2189 & 1.1946 & 0.9757 & 0.2190 & 1.1947 \\
\hline 1.40 & 0.9853 & 0.1699 & 1.1553 & 0.9854 & 0.1700 & 1.1554 \\
\hline 1.45 & 0.9926 & 0.1204 & 1.1131 & 0.9927 & 0.1205 & 1.1132 \\
\hline 1.50 & 0.9974 & 0.0706 & 1.0681 & 0.9975 & 0.0707 & 1.0682 \\
\hline
\end{tabular}



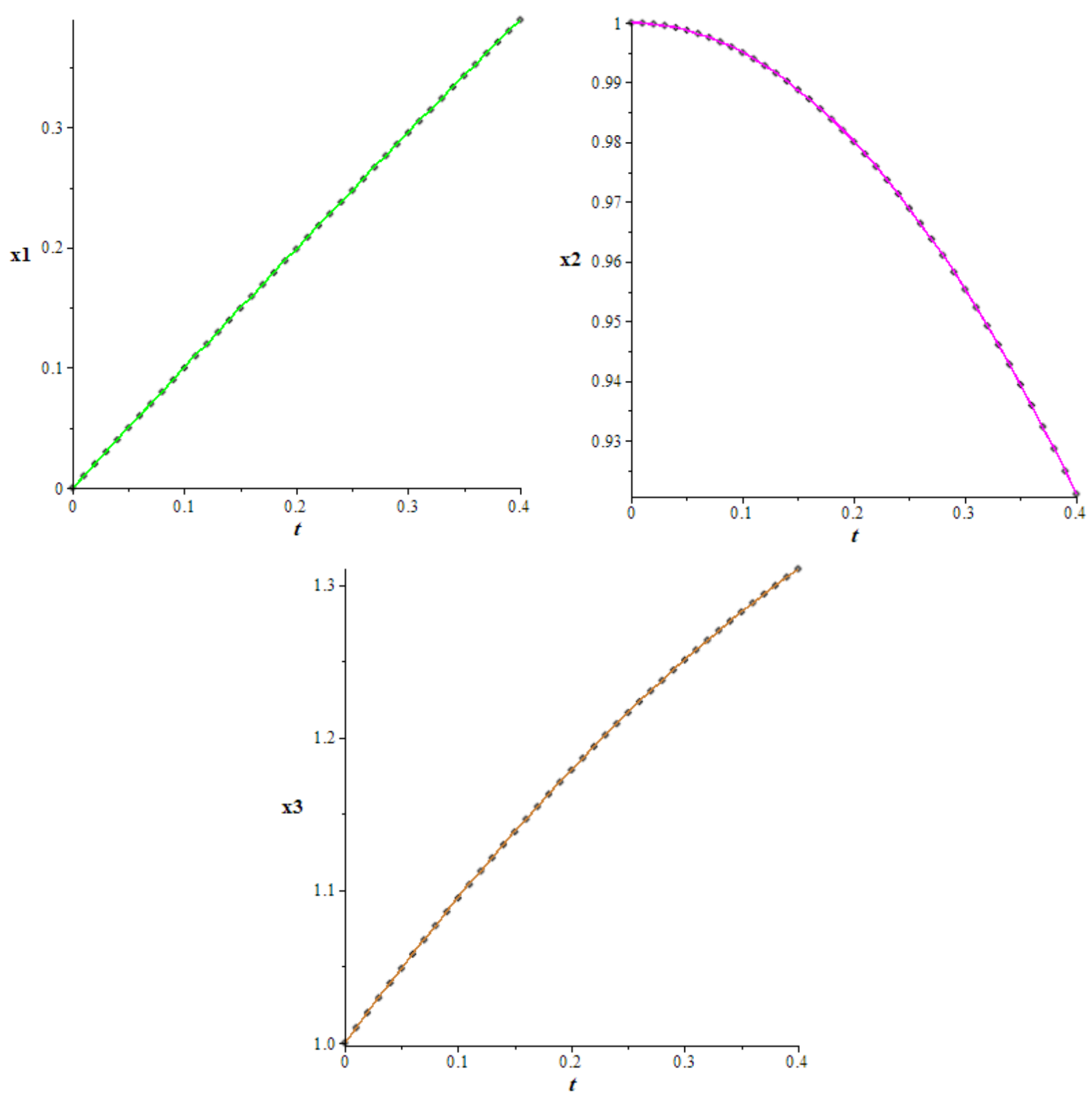

Fig. 2 The exact and approximate solutions result of systems of three Nonlinear Volterra integral equations for example 2.

Example3. Consider the system of Volterra integral equations

$$
\begin{gathered}
\mathrm{X}_{1}(t)=3 t+\cos 2 t-t^{3}+\int_{0}^{t}\left(\mathrm{X}_{1}(s)+2 \mathrm{X}_{2}(s)+3 \mathrm{X}_{3}(s)\right) d s \\
\mathrm{X}_{2}(t)=\frac{3}{2} \sin 2 t-t e^{t}+\int_{0}^{t}\left(t \mathrm{X}_{1}(s)+(2 \mathrm{t}-2 \mathrm{~s}) \mathrm{X}_{2}(s)\right) d s \\
\mathrm{X}_{3}(t)=t^{2}-e^{t}+\int_{0}^{t} \mathrm{X}_{1}(s) d s
\end{gathered}
$$

With the exact solution $\mathrm{X}_{1}(t)=e^{t}, \mathrm{X}_{2}(t)=\sin 2 t$ and $\mathrm{X}_{3}(t)=t^{2}-1$. 
Table.4 Numerical results and exact solution of systems of three Nonlinear Volterra integral equations for example 4.

\begin{tabular}{|c|c|c|c|c|c|c|}
\hline$t$ & $X_{1}(t)$ & $X_{2}(t)$ & $X_{3}(t)$ & $\begin{array}{l}\text { Exact1 } \\
=e^{t}\end{array}$ & $\begin{array}{l}\text { Exact2 } \\
=\sin 2 t\end{array}$ & $\begin{array}{l}\text { Exact } 3 \\
=t^{2}-1\end{array}$ \\
\hline 0.00 & 1.0000 & 0.0000 & -1.0000 & 1.0000 & 0.0000 & -1.0000 \\
\hline 0.05 & 1.0513 & 0.0998 & -0.9975 & 1.0513 & 0.0998 & -0.9975 \\
\hline 0.10 & 1.1052 & 0.1987 & -0.9900 & 1.1052 & 0.1987 & -0.9900 \\
\hline 0.15 & 1.1618 & 0.2955 & -0.9775 & 1.1618 & 0.2955 & -0.9775 \\
\hline 0.20 & 1.2214 & 0.3894 & -0.9600 & 1.2214 & 0.3894 & -0.9600 \\
\hline 0.25 & 1.2840 & 0.4794 & -0.9375 & 1.2840 & 0.4794 & -0.9375 \\
\hline 0.30 & 1.3499 & 0.5646 & -0.9100 & 1.3499 & 0.5646 & -0.9100 \\
\hline 0.35 & 1.4191 & 0.6442 & -0.8775 & 1.4191 & 0.6442 & -0.8775 \\
\hline 0.40 & 1.4918 & 0.7173 & -0.8400 & 1.4918 & 0.7174 & -0.8400 \\
\hline 0.45 & 1.5683 & 0.7833 & -0.7975 & 1.5683 & 0.7833 & -0.7975 \\
\hline 0.50 & 1.6487 & 0.8414 & -0.7500 & 1.6487 & 0.8415 & -0.7500 \\
\hline 0.55 & 1.7333 & 0.8912 & -0.6975 & 1.7333 & 0.8912 & -0.6975 \\
\hline 0.60 & 1.8221 & 0.9320 & -0.6400 & 1.8221 & 0.9320 & -0.6400 \\
\hline 0.65 & 1.9155 & 0.9635 & -0.5775 & 1.9155 & 0.9636 & -0.5775 \\
\hline 0.70 & 2.0138 & 0.9854 & -0.5100 & 2.0138 & 0.9854 & -0.5100 \\
\hline 0.75 & 2.1170 & 0.9975 & -0.4375 & 2.1170 & 0.9975 & -0.4375 \\
\hline 0.80 & 2.2255 & 0.9995 & -0.3600 & 2.2255 & 0.9996 & -0.3600 \\
\hline 0.85 & 2.3396 & 0.9916 & -0.2775 & 2.3396 & 0.9917 & -0.2775 \\
\hline 0.90 & 2.4596 & 0.9738 & -0.1900 & 2.4596 & 0.9738 & -0.1900 \\
\hline 0.95 & 2.5857 & 0.9463 & -0.0975 & 2.5857 & 0.9463 & -0.0975 \\
\hline 1.00 & 2.7183 & 0.9093 & 0.0000 & 2.7183 & 0.9093 & 0.0000 \\
\hline 1.05 & 2.8576 & 0.8632 & 0.1025 & 2.8577 & 0.8632 & 0.1025 \\
\hline 1.10 & 3.0042 & 0.8084 & 0.2100 & 3.0042 & 0.8085 & 0.2100 \\
\hline 1.15 & 3.1582 & 0.7457 & 0.3225 & 3.1582 & 0.7457 & 0.3225 \\
\hline 1.20 & 3.3201 & 0.6754 & 0.4400 & 3.3201 & 0.6755 & 0.4400 \\
\hline 1.25 & 3.4903 & 0.5984 & 0.5625 & 3.4903 & 0.5985 & 0.5625 \\
\hline 1.30 & 3.6693 & 0.5154 & 0.6900 & 3.6693 & 0.5155 & 0.6900 \\
\hline 1.35 & 3.8574 & 0.4273 & 0.8225 & 3.8574 & 0.4274 & 0.8225 \\
\hline 1.40 & 4.0552 & 0.3349 & 0.9600 & 4.0552 & 0.3350 & 0.9600 \\
\hline 1.45 & 4.2631 & 0.2392 & 1.1025 & 4.2631 & 0.2392 & 1.1025 \\
\hline 1.50 & 4.4817 & 0.1411 & 1.2500 & 4.4817 & 0.1411 & 1.2500 \\
\hline
\end{tabular}



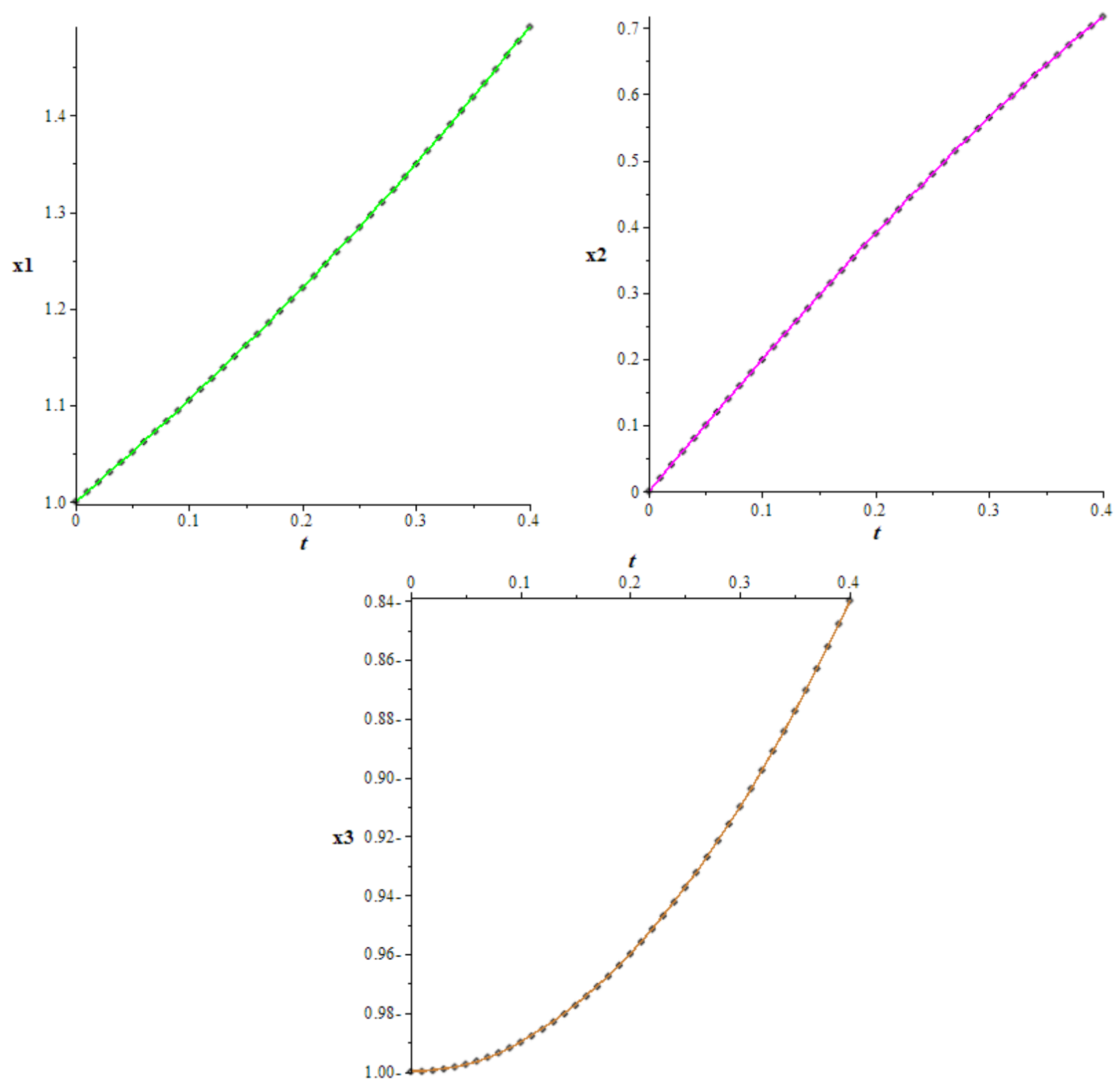

Fig. 3 The exact and approximate solutions result of systems of three Nonlinear Volterra integral equations for example 3.

Example4. Consider the system of Volterra integral equations

$$
\begin{gathered}
\mathrm{X}_{1}(t)=t-\frac{1}{12} t^{4}-\frac{1}{20} t^{5}+\int_{0}^{t}\left((\mathrm{t}-\mathrm{s}) \mathrm{X}_{2}(s)+(\mathrm{t}-\mathrm{s}) \mathrm{X}_{3}(s)\right) d s \\
\mathrm{X}_{2}(t)=t^{2}-\frac{1}{6} t^{3}-\frac{1}{20} t^{5}+\int_{0}^{t}\left((\mathrm{t}-\mathrm{s}) \mathrm{X}_{1}(\mathrm{~s})+(\mathrm{t}-\mathrm{s}) \mathrm{X}_{3}(\mathrm{~s})\right) d s \\
\mathrm{X}_{3}(t)=\frac{5}{6} t^{3}-\frac{1}{12} t^{4}+\int_{0}^{t}\left((\mathrm{t}-\mathrm{s}) \mathrm{X}_{1}(\mathrm{~s})+(\mathrm{t}-\mathrm{s}) \mathrm{X}_{2}(s)\right) d s
\end{gathered}
$$

With the exact solution $\mathrm{X}_{1}(t)=t, \mathrm{X}_{2}(t)=t^{2}$ and $\mathrm{X}_{3}(t)=t^{3}$. 
Table.4 Numerical results and exact solution of systems of three Nonlinear Volterra integral equations for example 4.

\begin{tabular}{|c|c|c|c|c|c|c|}
\hline$t$ & $X_{1}(t)$ & $X_{2}(t)$ & $X_{3}(t)$ & $\begin{array}{l}\text { Exact1 } \\
=t\end{array}$ & $\begin{array}{l}\text { Exact2 } \\
=t^{2}\end{array}$ & $\begin{array}{l}\text { Exact } 3 \\
=t^{3}\end{array}$ \\
\hline 0.00 & 0.0000 & 0.0000 & 0.0000 & 0.0000 & 0.0000 & 0.0000 \\
\hline 0.05 & 0.0500 & 0.0025 & 0.0001 & 0.0500 & 0.0025 & 0.0001 \\
\hline 0.10 & 0.1000 & 0.0100 & 0.0010 & 0.1000 & 0.0100 & 0.0010 \\
\hline 0.15 & 0.1500 & 0.0225 & 0.0034 & 0.1500 & 0.0225 & 0.0034 \\
\hline 0.20 & 0.2000 & 0.0400 & 0.0080 & 0.2000 & 0.0400 & 0.0080 \\
\hline 0.25 & 0.2500 & 0.0625 & 0.0156 & 0.2500 & 0.0625 & 0.0156 \\
\hline 0.30 & 0.3000 & 0.0900 & 0.0270 & 0.3000 & 0.0900 & 0.0270 \\
\hline 0.35 & 0.3500 & 0.1225 & 0.0429 & 0.3500 & 0.1225 & 0.0429 \\
\hline 0.40 & 0.4000 & 0.1600 & 0.0640 & 0.4000 & 0.1600 & 0.0640 \\
\hline 0.45 & 0.4500 & 0.2025 & 0.0911 & 0.4500 & 0.2025 & 0.0911 \\
\hline 0.50 & 0.5000 & 0.2500 & 0.1250 & 0.5000 & 0.2500 & 0.1250 \\
\hline 0.55 & 0.5500 & 0.3025 & 0.1664 & 0.5500 & 0.3025 & 0.1664 \\
\hline 0.60 & 0.6000 & 0.3600 & 0.2160 & 0.6000 & 0.3600 & 0.2160 \\
\hline 0.65 & 0.6500 & 0.4225 & 0.2746 & 0.6500 & 0.4225 & 0.2746 \\
\hline 0.70 & 0.7000 & 0.4900 & 0.3430 & 0.7000 & 0.4900 & 0.3430 \\
\hline 0.75 & 0.7500 & 0.5625 & 0.4219 & 0.7500 & 0.5625 & 0.4219 \\
\hline 0.80 & 0.8000 & 0.6400 & 0.5120 & 0.8000 & 0.6400 & 0.5120 \\
\hline 0.85 & 0.8500 & 0.7225 & 0.6141 & 0.8500 & 0.7225 & 0.6141 \\
\hline 0.90 & 0.9000 & 0.8100 & 0.7290 & 0.9000 & 0.8100 & 0.7290 \\
\hline 0.95 & 0.9500 & 0.9025 & 0.8573 & 0.9500 & 0.9025 & 0.8574 \\
\hline 1.00 & 1.0000 & 1.0000 & 1.0000 & 1.0000 & 1.0000 & 1.0000 \\
\hline 1.05 & 1.0500 & 1.1025 & 1.1576 & 1.0500 & 1.1025 & 1.1576 \\
\hline 1.10 & 1.1000 & 1.2100 & 1.3310 & 1.1000 & 1.2100 & 1.3310 \\
\hline 1.15 & 1.1500 & 1.3225 & 1.5208 & 1.1500 & 1.3225 & 1.5209 \\
\hline 1.20 & 1.2000 & 1.4400 & 1.7280 & 1.2000 & 1.4400 & 1.7280 \\
\hline 1.25 & 1.2500 & 1.5625 & 1.9531 & 1.2500 & 1.5625 & 1.9531 \\
\hline 1.30 & 1.3000 & 1.6899 & 2.1970 & 1.3000 & 1.6900 & 2.1970 \\
\hline 1.35 & 1.3499 & 1.8224 & 2.4603 & 1.3500 & 1.8225 & 2.4604 \\
\hline 1.40 & 1.3999 & 1.9599 & 2.7439 & 1.4000 & 1.9600 & 2.7440 \\
\hline 1.45 & 1.4499 & 2.1024 & 3.0486 & 1.4500 & 2.1025 & 3.0486 \\
\hline 1.50 & 1.4999 & 2.2499 & 3.3749 & 1.5000 & 2.2500 & 3.3750 \\
\hline
\end{tabular}



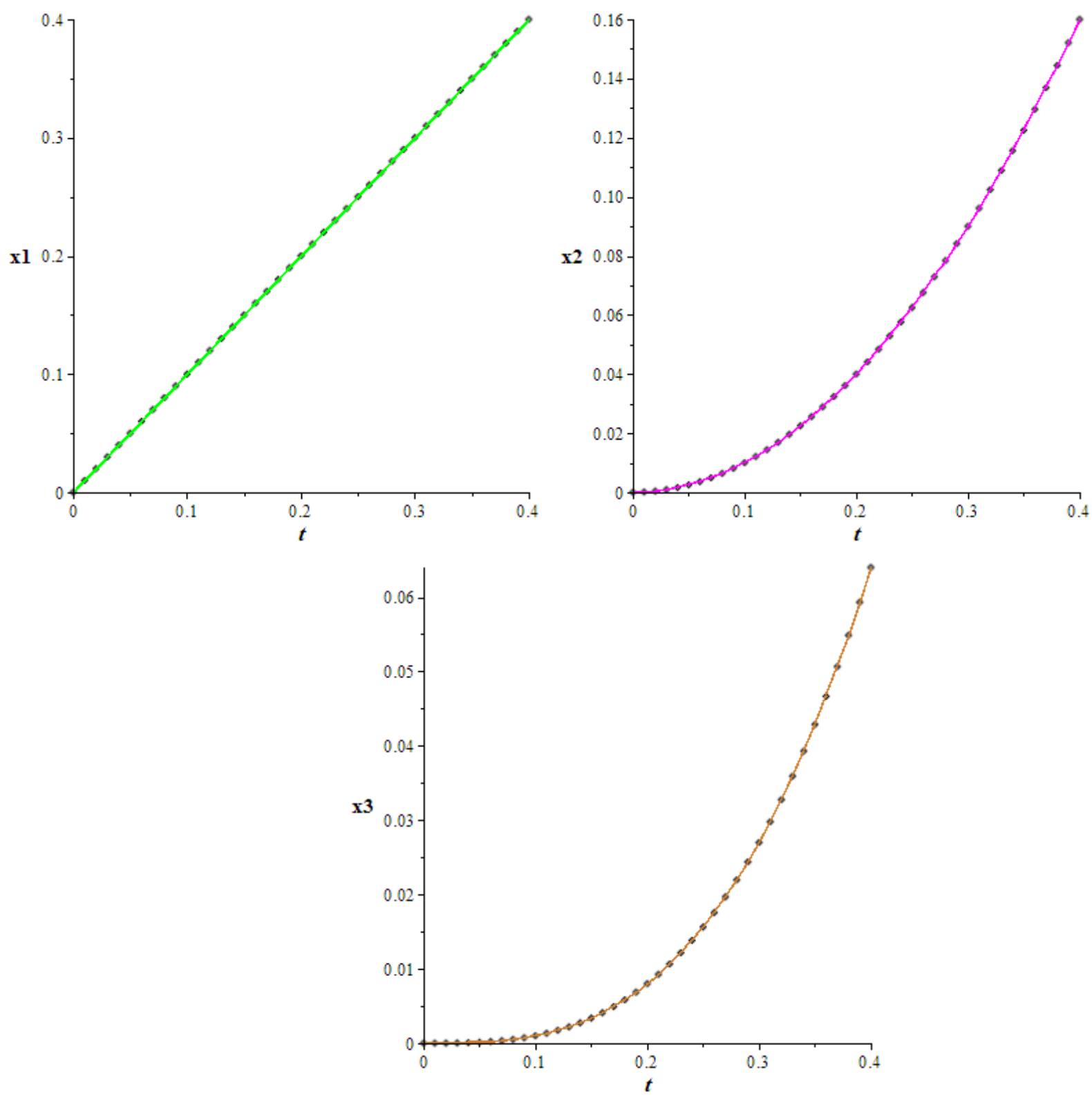

Fig. 4 The exact and approximate solutions result of systems of three Nonlinear Volterra integral equations for example 4.

Example5. Consider the system of Volterra integral equations

$$
\begin{gathered}
\mathrm{X}_{1}(t)=\cos t-2 t^{2} \cos t+4 \int_{0}^{t} \mathrm{X}_{1}(s) \mathrm{X}_{2}(s) d s \\
\mathrm{X}_{2}(t)=t-\frac{1}{6} t^{6} \cos t+\frac{1}{36} t^{6}+\frac{1}{3} \int_{0}^{t} s \mathrm{X}_{3}^{2}(s) \mathrm{X}_{1}(s) d s \\
\mathrm{X}_{3}(t)=t^{2}+\frac{1}{15} t^{5}+\int_{0}^{t} \mathrm{sX}_{3}(s) \mathrm{X}_{2}(s) d s
\end{gathered}
$$


With the exact solution $\mathrm{X}_{1}(t)=\cos t, \mathrm{X}_{2}(t)=t$ and $\mathrm{X}_{3}(t)=t^{2}$.

Table.5 Numerical results and exact solution of systemsof three Nonlinear Volterra integral equations for example 5.

\begin{tabular}{|c|c|c|c|c|c|c|}
\hline$t$ & $X_{1}(t)$ & $X_{2}(t)$ & $X_{3}(t)$ & $\begin{array}{l}\text { Exact1 } \\
=\cos t\end{array}$ & $\begin{array}{l}\text { Exact2 } \\
=t\end{array}$ & $\begin{array}{l}\text { Exact3 } \\
=t^{2}\end{array}$ \\
\hline 0.000 & 1.0000 & 0.0000 & 0.0000 & 1.0000 & 0.0000 & 0.0000 \\
\hline 0.005 & 1.0000 & 0.0050 & 0.0000 & 1.0000 & 0.0050 & 0.0000 \\
\hline 0.010 & 1.0000 & 0.0100 & 0.0001 & 0.9998 & 0.0100 & 0.0001 \\
\hline 0.015 & 0.9999 & 0.0150 & 0.0002 & 0.9996 & 0.0150 & 0.0002 \\
\hline 0.020 & 0.9998 & 0.0200 & 0.0004 & 0.9992 & 0.0200 & 0.0004 \\
\hline 0.025 & 0.9997 & 0.0250 & 0.0006 & 0.9988 & 0.0250 & 0.0006 \\
\hline 0.030 & 0.9996 & 0.0300 & 0.0009 & 0.9982 & 0.0300 & 0.0009 \\
\hline 0.035 & 0.9994 & 0.0350 & 0.0012 & 0.9976 & 0.0350 & 0.0012 \\
\hline 0.040 & 0.9992 & 0.0400 & 0.0016 & 0.9968 & 0.0400 & 0.0016 \\
\hline 0.045 & 0.9990 & 0.0450 & 0.0020 & 0.9960 & 0.0450 & 0.0020 \\
\hline 0.050 & 0.9988 & 0.0500 & 0.0025 & 0.9950 & 0.0500 & 0.0025 \\
\hline 0.055 & 0.9985 & 0.0550 & 0.0030 & 0.9940 & 0.0550 & 0.0030 \\
\hline 0.060 & 0.9982 & 0.0600 & 0.0036 & 0.9928 & 0.0600 & 0.0036 \\
\hline 0.065 & 0.9979 & 0.0650 & 0.0042 & 0.9916 & 0.0650 & 0.0042 \\
\hline 0.070 & 0.9976 & 0.0700 & 0.0049 & 0.9902 & 0.0700 & 0.0049 \\
\hline 0.075 & 0.9972 & 0.0750 & 0.0056 & 0.9888 & 0.0750 & 0.0056 \\
\hline 0.080 & 0.9968 & 0.0800 & 0.0064 & 0.9872 & 0.0800 & 0.0064 \\
\hline 0.085 & 0.9964 & 0.0850 & 0.0072 & 0.9856 & 0.0850 & 0.0072 \\
\hline 0.090 & 0.9960 & 0.0900 & 0.0081 & 0.9838 & 0.0900 & 0.0081 \\
\hline 0.095 & 0.9955 & 0.0950 & 0.0090 & 0.9820 & 0.0950 & 0.0090 \\
\hline 0.100 & 0.9951 & 0.1000 & 0.0100 & 0.9801 & 0.1000 & 0.0100 \\
\hline 0.105 & 0.9946 & 0.1050 & 0.0110 & 0.9780 & 0.1050 & 0.0110 \\
\hline 0.110 & 0.9940 & 0.1100 & 0.0121 & 0.9759 & 0.1100 & 0.0121 \\
\hline 0.115 & 0.9935 & 0.1150 & 0.0132 & 0.9737 & 0.1150 & 0.0132 \\
\hline 0.120 & 0.9929 & 0.1200 & 0.0144 & 0.9713 & 0.1200 & 0.0144 \\
\hline 0.125 & 0.9923 & 0.1250 & 0.0156 & 0.9689 & 0.1250 & 0.0156 \\
\hline 0.130 & 0.9917 & 0.1300 & 0.0169 & 0.9664 & 0.1300 & 0.0169 \\
\hline 0.135 & 0.9911 & 0.1350 & 0.0182 & 0.9638 & 0.1350 & 0.0182 \\
\hline 0.140 & 0.9904 & 0.1400 & 0.0196 & 0.9611 & 0.1400 & 0.0196 \\
\hline 0.145 & 0.9897 & 0.1450 & 0.0210 & 0.9582 & 0.1450 & 0.0210 \\
\hline 0.150 & 0.9890 & 0.1500 & 0.0225 & 0.9553 & 0.1500 & 0.0225 \\
\hline
\end{tabular}



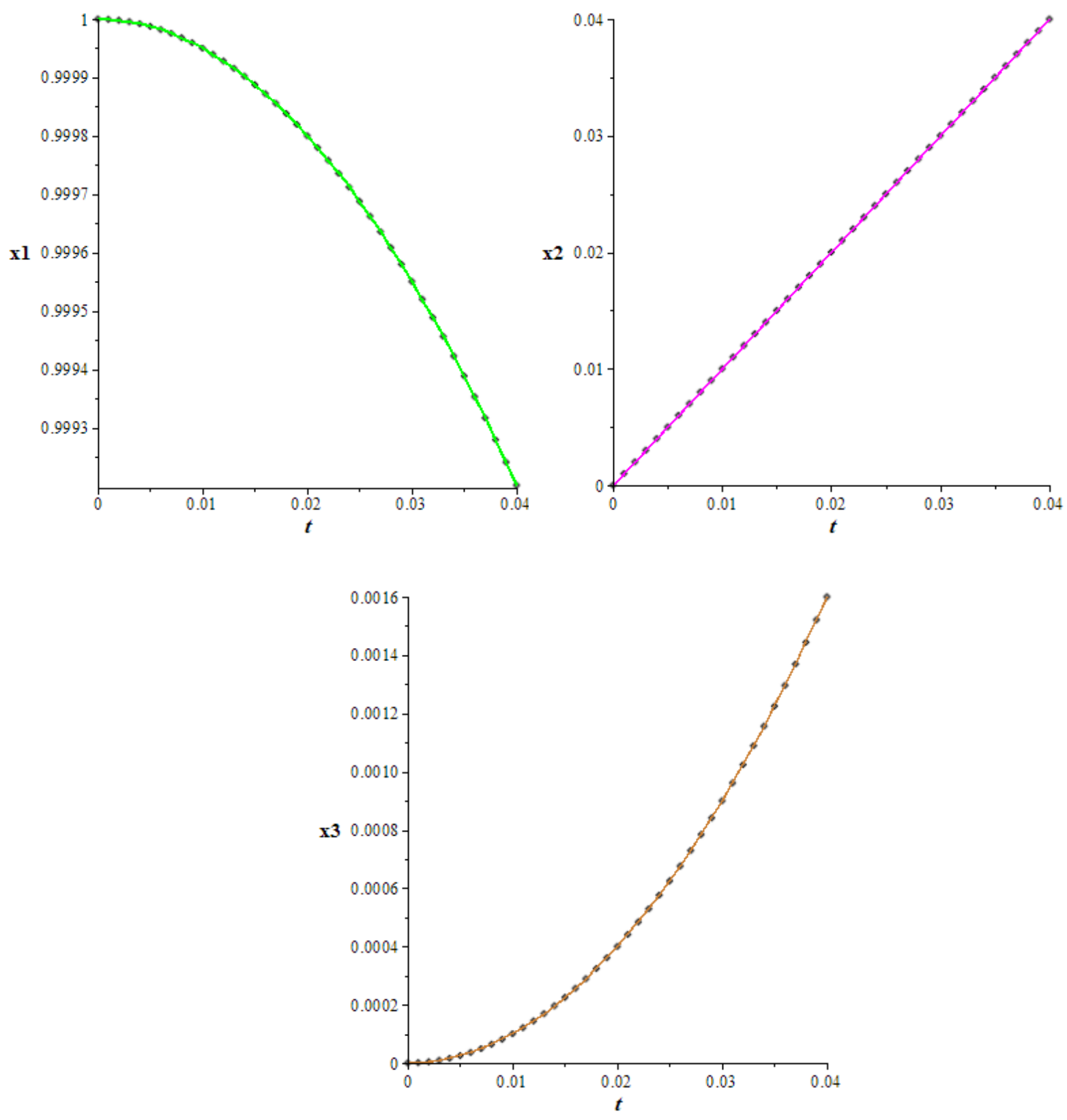

Fig. 5 The exact and approximate solutions result of systems of three Nonlinear Volterra integral equations for example 5.

\section{Conclusion}

In this project, we have studied system of three nonlinear Volterra integral equations with the implicit trapezoidal method. The basic goal of the present project is to mechanize the computing process of our implicit trapezoidal method by a Maple program and obtain more precise values of the solutions. The results showed that the implicit trapezoidal method is remarkably effective and performing is very easy. The computed values and graphics, illustrated by the results, agree well with the exact solution.

\section{Acknowledgments}

This project was funded by the Deanship of Scientific Research (DSR), King Abdulaziz University, under grant no. (446-363-1436/HiCi). The authors, therefore, acknowledge with thanks DSR technical and financial support. 


\section{References}

Balakumar, V., \& Murugesan, K. (2013). Numerical Solution of Systems of Linear Volterra Integral Equations Using Block-Pulse Functions. Malaya Journal of Matematik, S(1), 77-84.

Becker, L. C., \& Wheeler, M. (2005). Numerical and Graphical Solutions of Volterra Equations of the Second Kind, Maple Application Center.

Berenguer, M. I., Gamez, D., Garralda-Guillem, A. I., Ruiz Galan, M., \& Serrano Perez, M. C. (2011). Biorthogonal Systems for Solving Volterra Integral equation Systems of the Second Kind. Journal of Computional and Applied Mathematics, 235(7), 1875-1883. https://doi.org/10.1016/j.cam.2010.07.011

Biazar, J., \& Eslami, M. (2011). Modified HPM for Solving Systems of Volterra Integral Equation of the Second Kind. Journal of King Saud University-Science, 23(1), 35-39. https://doi.org/10.1016/j.jksus.2010.06.004

Burden, R. L, \& Douglas Faires, J. (2005) .Numerical Analysis, 8th ed., Thomson Brooks/Cole, Belmont, CA.

Burton,T. A. (2005). Volterra Integral and Differential Equations, 2nd ed. Mathematics in Science \& Engineering, 202, Elsevier.

Effati, S., \& NooriSkandari, M. H. (2012). Optimal Control Approach for Solving Linear Volterra Integral Equations. I.J.Intelligent Systems and Applications, 4, 40-46. https://doi.org/10.5815/ijisa.2012.04.06

Linz, P. (1985). Analytical and Numerical Methods for Volterra Equations. Studies in Applied Mathematics 7, SIAM, Philadelphia. https://doi.org/10.1137/1.9781611970852

Maturi, D. A. (2014). Numerical Solution of System of Two Nonlinear Volterra Integral Equations. International Journal of Computers \& Technology, 12(10), 3967-3975.

Maturi, D. A., Bajamal, A. Z., \& AlGethami, B. M. (2014). Numerical Solution of Volterra Integral Equation of Second Kind Using Implicit Trapezoial. Journal of Advances In Mathematics, 8(2), 1540-1553.

Maturi, Dalal A. (2014). Application of Adomian Decomposition Method for Solving of Fredholm Integral Equation of the Second Kind. European Journal of Science and Engineering, 9(2), 1-9.

Maturi, Dalal A. (2014). Adomian Decomposition Method of Fredholm Integral Equation of the Second Kind Using Maple. Journal of Advances In Mathematics, 9(1), 1868-1875.

Mirzaee, F. (2010). Numerical Computional Solution of the Linear Volterra Integral Equations System Via Rationalized Haar Functions. Journal of King Saud University- Science, 22(4), 265-268. https://doi.org/10.1016/j.jksus.2010.05.010

Wazwaz, A. M. (2011). Linear and Nonlinear Integral Equation: Methods and Applications, Springer. https://doi.org/10.1007/978-3-642-21449-3

\section{Copyrights}

Copyright for this article is retained by the author(s), with first publication rights granted to the journal.

This is an open-access article distributed under the terms and conditions of the Creative Commons Attribution license (http://creativecommons.org/licenses/by/4.0/). 